\title{
Post-Ugi gold-catalyzed diastereoselective domino cyclization for the synthesis of diversely substituted spiroindolines
}

\author{
Amit Kumar ${ }^{1,2}$, Dipak D. Vachhani ${ }^{1}$, Sachin G. Modha ${ }^{* 1,3}$, Sunil K. Sharma ${ }^{2}$, \\ Virinder S. Parmar ${ }^{2}$ and Erik V. Van der Eycken ${ }^{* 1}$
}

\section{Full Research Paper}

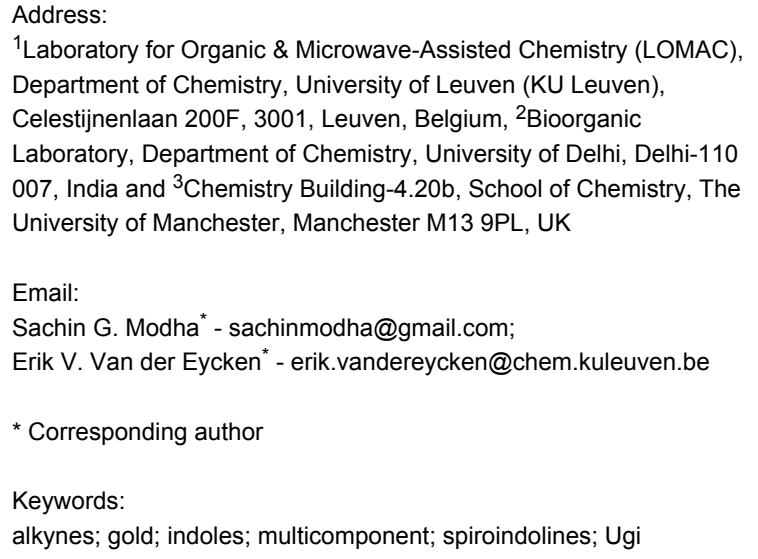

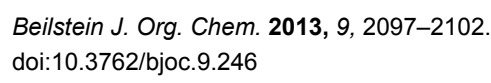

\begin{abstract}
An Ugi four-component reaction of propargylamine with 3-formylindole and various acids and isonitriles produces adducts which are subjected to a cationic gold-catalyzed diastereoselective domino cyclization to furnish diversely substituted spiroindolines. All the reactions run via an exo-dig attack in the hydroarylation step followed by an intramolecular diastereoselective trapping of the imminium ion. The whole sequence is atom economic and the application of a multicomponent reaction assures diversity.
\end{abstract}

\section{Introduction}

The importance of nitrogen containing heterocyclic molecules in chemical biology is undisputed. The synthesis of such biologically interesting heterocycles is generally target-oriented, inspired by nature or randomly directed. In all these cases the design of a synthetic sequence to produce a library of diversely substituted molecules is the first and most important step. The basic concept of diversity-oriented synthesis (DOS) involves short reaction sequences, a strong focus on bond construction, and functional group compatibility [1-3]. Reactions that involve multiple bond formation, such as multicomponent reactions [4-9] and tandem reactions [10-16], are very useful in this context.

As an efficient activator of alkynes, gold has recently attracted a lot of attention [17-36]. Many tandem approaches have been reported which utilize this coinage metal for the construction of variously substituted complex molecules [37-43]. We have recently reported a post-Ugi gold-catalyzed intramolecular 
domino cyclization sequence which produces spiroindolines (Scheme 1) [44]. The first step in this sequence is an Ugi fourcomponent reaction (Ugi-4CR) [4,5] with 2-alkynoic acid as an alkyne source. The second step is a cationic gold-catalyzed intramolecular hydroarylation tandem cyclization to produce spiroindolines with complete diastereoselectivity. This synthetic sequence is atom economic and mild conditions are applied to generate a very complex molecular structure from readily available starting materials. Based on this work and our continuous interest in transition metal catalysis [45-54], multicomponent reactions [55-57] and the chemistry of the indole core [58-60], we herein report a post-Ugi gold-catalyzed intramolecular domino cyclization sequence for the synthesis of spiroindolines with propargylamines as an alkyne source (Scheme 1).

\section{Results and Discussion}

The use of benzoic acid as an acid component in the Ugi-4CR did not produce the Ugi-adduct in good yield even after a prolonged reaction time. Therefore, we switched to phenylacetic acid. The Ugi-4CR of indole-3-carboxaldehyde (1a), propargylamine (2a), phenylacetic acid (3a) and tert-butylisonitrile (4a) in methanol at $50{ }^{\circ} \mathrm{C}$ gave the Ugi-adduct 5a with an excellent yield of $94 \%$. With compound $\mathbf{5 a}$ in hand we were keen to apply the previously developed conditions for intramolecular hydroarylation [44]. Reaction of 5a with $5 \mathrm{~mol} \%$ of $\mathrm{Au}\left(\mathrm{PPh}_{3}\right) \mathrm{SbF}_{6}$ in chloroform at room temperature produced the desired spiroindoline $6 \mathrm{a}$ in a moderate yield of 55\% along with some unidentified byproducts (Table 1 , entry 1 ). The use of a protic acid with a gold catalyst is known in the literature [6164]. To our delight, when the above reaction was carried out with 1 equivalent of trifluoroacetic acid (TFA) the yield was improved to $81 \%$ (Table 1, entry 2). Apart from being a good proton source TFA might be working as a coligand.

Experiments with $\mathrm{PtCl}_{2}$ as a catalyst did not show any conversion and the starting material was recovered quantitatively (Table 1, entries 3 and 4). In absence of the gold catalyst no product could be observed (Table 1, entry 5). The application of $p$-toluenesulfonic acid (PTSA) instead of TFA did not improve the outcome (Table 1 , entry 6 ).

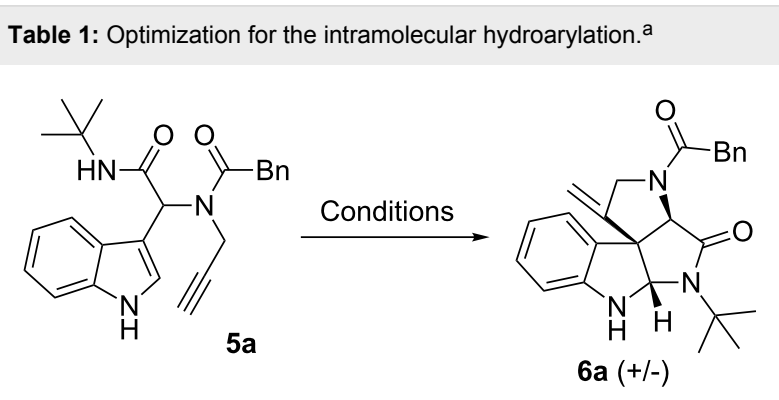

\begin{tabular}{lllll}
\hline Entry & Catalyst (mol \%) & Acid (1 equiv) & Time h & \% Yield \\
\hline 1 & $\mathrm{Au}\left(\mathrm{PPh}_{3}\right) \mathrm{SbF}_{6}(5)$ & - & 2 & $55^{\mathrm{c}}$ \\
2 & $\mathbf{A u}\left(\mathrm{PPh}_{3}\right) \mathrm{SbF}_{6}(5)$ & TFA & $\mathbf{2}$ & $\mathbf{8 1}$ \\
3 & $\mathrm{PtCl}_{2}(5)$ & - & 10 & $-{ }^{\mathrm{d}}$ \\
4 & $\mathrm{PtCl}_{2}(5)$ & TFA & 10 & - $^{\mathrm{d}}$ \\
5 & - & TFA & 10 & - $^{\mathrm{d}}$ \\
6 & $\mathrm{Au}\left(\mathrm{PPh}_{3}\right) \mathrm{SbF}_{6}(5)$ & $\mathrm{PTSA}$ & 2 & 70 \\
\hline
\end{tabular}

aAll the reactions were run on $0.1 \mathrm{mmol}$ scale of $5 \mathbf{a}$ with chloroform $(2 \mathrm{~mL})$ as a solvent at rt. ${ }^{\text {I }}$ solated yields. CUnidentified byproducts were formed. ${ }^{\mathrm{d}}$ No conversion.

\footnotetext{
Scheme 1: Gold-catalyzed approaches towards spiroindolines.
} 
Having the optimized conditions in hand (Table 1, entry 2), various Ugi-adducts $\mathbf{5} \mathbf{b}-\mathbf{q}$ were synthesized and subjected to this hydroarylation domino cyclization sequence (Table 2). Different substituents are well-tolerated and the sprioindolines were obtained in good to excellent yields. A methyl substituent on the indole nitrogen did not hamper the domino cyclization (Table 2, entries 4, 6, 11, 12, 14, 15). Substituents like tertbutyl, cyclohexyl and $n$-butyl on the isonitrile are well-tolerated for the domino cyclization on the second position of the indole (Table 2, entries 1-16). Regarding the substituents coming from the acid part, tert-butyl gave a decreased yield probably due to steric hindrance (Table 2, entry 5). It is noteworthy that the gold-catalyzed intramolecular hydroarylation exclusively gives the exo-dig product in all cases and with complete diastereoselectivity.

A plausible mechanism [30,44] is shown in Scheme 2 with only the $R$-isomer of the Ugi-adduct 5a to simplify the discussion. The cationic gold coordinates with the terminal alkyne which becomes activated for a nucleophilic attack. This can occur from both sides of the indole core. When the attack occurs from the back side of the indole core, spiro intermediate $\mathbf{B}$

Table 2: Scope and limitations of intramolecular domino cyclization. ${ }^{a}$<smiles>C#CCN(C(=O)Cc1ccccc1)C(C(=O)NC1CCCCC1)c1c[nH]c2ccccc12</smiles>

5b, $87 \%$<smiles>C#CCN(C(=O)Cc1ccccc1)C(C(=O)NC(C)(C)C)c1c[nH]c2c(C)cccc12</smiles>

5c, $86 \%$<smiles>C#CCN(C(=O)[NH2+]C(C)(C)C)C(C(=O)NC1CCCCC1)c1c[nH]c2c(C)cccc12</smiles>

5d, $69 \%$<smiles>C#CCN(C(=O)[NH2+]C(C)(C)C)C(C(=O)NC(C)(C)C)c1cn(C)c2ccccc12</smiles>

5e, $72 \%$<smiles>C#CCN(C(=O)C(C)(C)C)C(C(=O)NC1CCCCC1)c1c[nH]c2ccccc12</smiles>

5f, $68 \%$

Spiroindolines 6 (+/-) Entry

Ugi adduct 5

Spiroindolines $6(+/-)$<smiles>CC1CN(C(=O)Cc2ccccc2)[C@H]2C(=O)N(C3CCCCC3)[C@H]3Nc4ccccc4C123</smiles>

6b, $70 \%$<smiles>Cc1ccccc1N[C@H]1N(C(C)(C)C)C(=O)[C@@H]2N(C(=O)Cc3ccccc3)CC3CC[C@]12[C@H]3C</smiles>

6c, $60 \%$<smiles>Cc1cccc2c1NC1CC23C(C)CN(C(=O)P(C)C)[C@H]3C(=O)N1C1CCCCC1</smiles>

6d, $76 \%$

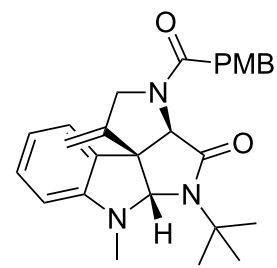

$6 e, 66 \%$<smiles></smiles>

9<smiles>C#CCN(C(=O)[N+](C)(C)C)C(C(=O)NC1CCCCC1)c1c[nH]c2ccccc12</smiles>

5j, $89 \%$<smiles>C#CCN(C(=O)Cc1ccccc1)C(C(=O)NCc1ccccc1)c1c[nH]c2ccccc12</smiles>

5k, $63 \%$<smiles>C#CCN(C(=O)Cc1ccccc1)C(C(=O)NC1CCCCC1)c1cn(C)c2ccccc12</smiles>

5I, $77 \%$<smiles>C#CCN(C(=O)[R16](C)(C)C)C(C(=O)NC1CCCCC1)c1cn(C)c2ccccc12</smiles>

5m, $74 \%$<smiles>C#CCN(C(=O)CCc1ccccc1)C(C(=O)NC1CCCCC1)c1c[nH]c2ccccc12</smiles><smiles>CCCC12CC3Cc4ccccc4NC1N(C(=O)N1CCCCC1)C(=O)C32</smiles>

6j, $75 \%$<smiles>CC1CN(C(=O)Cc2ccccc2)[C@H]2C(=O)N(Cc3ccccc3)[C@H]3Nc4ccccc4C132</smiles>

6k, $80 \%$<smiles>CN1c2ccccc2C23CCC2CN(C(=O)Cc2ccccc2)C3C(=O)N1C1CCCCC1</smiles>

6I, $74 \%$

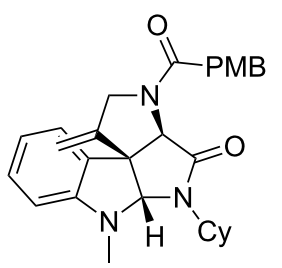

$6 \mathrm{~m}, 68 \%$<smiles>O=C1[C@H]2N(C(=O)CCc3ccccc3)CCCC23c2ccccc2NC3N1C1CCCCC1</smiles>

6n, $72 \%$ 
Table 2: Scope and limitations of intramolecular domino cyclization. ${ }^{\text {a }}$ (continued)

6<smiles>C#CCN(C(=O)Cc1ccccc1)C(C(=O)NC(C)(C)C)c1cn(C)c2ccccc12</smiles>

$5 g, 77 \%$<smiles>C#CCN(C(=O)CCc1ccccc1)C(C(=O)NC(C)(C)C)c1c[nH]c2ccccc12</smiles>

5h, $79 \%$

8

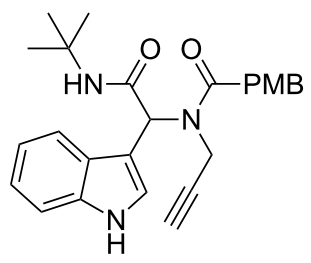

5i, $67 \%$<smiles>CN1c2ccccc2C2C3CN(C(=O)Cc4ccccc4)C(C(=O)N1C(C)(C)C)C32</smiles>

6g, $71 \%$<smiles>CC1CN(C(=O)CCc2ccccc2)[C@H]2C(=O)N(C(C)(C)C)[C@H]3Nc4ccccc4[C@@]123</smiles>

$6 h, 83 \%$<smiles>[R15]C(=O)N1CC(=O)[C@@]23C(=O)N(C(C)(C)C)C(=O)[C@@]1([CH])[C@@H]2Nc1ccccc13</smiles>

6i, 69\%<smiles>C#CCN(C(=O)CCc1ccccc1)C(C(=O)NC(C)(C)C)c1cn(C)c2ccccc12</smiles>

5o, $68 \%$<smiles>C#CCN(C(=O)CCc1ccccc1)C(C(=O)NC1CCCCC1)c1cn(C)c2ccccc12</smiles>

5p, 58\%<smiles>[R15]C(=O)N(CC#C)C(C(=O)NCc1ccccc1)c1c[nH]c2ccccc12</smiles>

5q, $59 \%$<smiles>CC1C(=O)N(C(C)(C)C)C2CCN(C(=O)CCc3ccccc3)C3C(=O)N(C)c4ccccc4C132</smiles>

6o, $60 \%$<smiles>CC1CN(C(=O)CCc2ccccc2)[C@@H]2C(=O)N(C3CCCCC3)[C@H]1[C@@]21c2ccccc2N(C)C1C</smiles>

6 p, $84 \%$

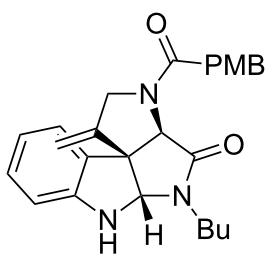

6q, $69 \%$

${ }^{a} A l l$ the reaction were run on a $0.2 \mathrm{mmol}$ scale of $\mathbf{5}$ in a screw capped vial employing the optimal conditions of Table 1. Cy $=$ cyclohexyl, Bn $=$ benzyl, $\mathrm{PMB}=p$-methoxybenzyl, $\mathrm{Bu}=n$-butyl.

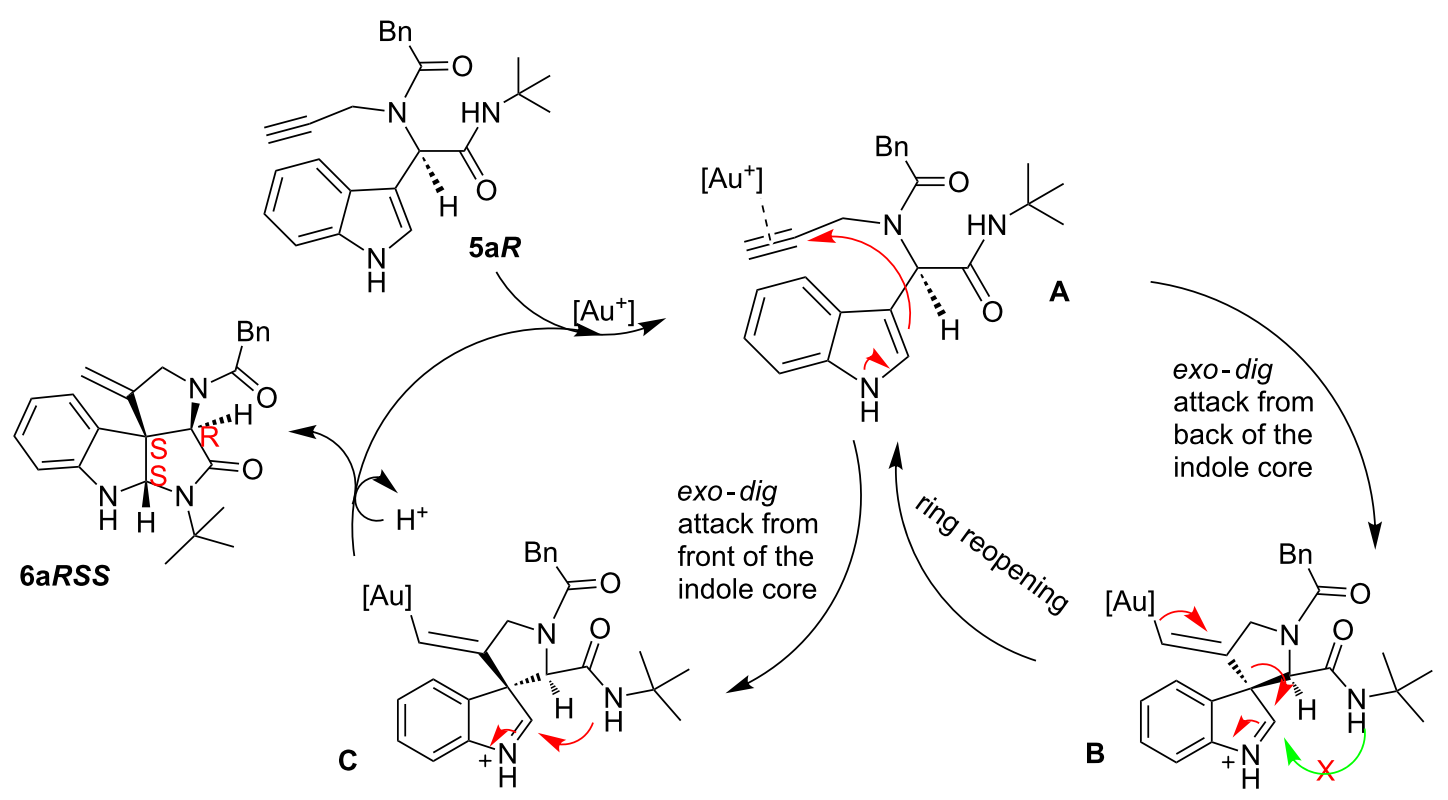

Scheme 2: Plausible mechanism for the domino sequence.

will be formed. However, in this spiro intermediate the intramolecular trapping of the imminium ion by the amidic $\mathrm{NH}$ is sterically impossible and thus the intermediate reopens to inter- mediate A. If the attack takes place from the front side of the indole core, intermediate $\mathbf{C}$ is formed and trapping is possible. After deprotonation and protodeauration the desired spiro- 
indoline 6a is formed with the stereochemistry of two new stereocenters $S$.

\section{Conclusion}

In conclusion we have developed a diversity-oriented post-Ugi gold-catalyzed intramolecular hydroarylation domino cyclization sequence for the diastereoselective synthesis of spiroindolines. The mild reaction conditions and short synthetic sequence are the merits of this method. The flexibility given by the multicomponent reaction assures the generation of diversity.

\section{Experimental}

\section{General procedure for the synthesis of spiroindolines $\mathbf{6 a}-\mathbf{q}$}

To a screw capped vial $\mathrm{Au}\left(\mathrm{PPh}_{3}\right) \mathrm{Cl}(5 \mathrm{~mol} \%)$ and $\mathrm{AgSbF}_{6}$ ( $5 \mathrm{~mol} \%$ ) were loaded along with chloroform $(2 \mathrm{~mL})$. Ugi product $5(0.2 \mathrm{mmol})$ was added followed by TFA (1 equiv), and the reaction mixture was stirred at $\mathrm{rt}$. After completion, the reaction mixture was partitioned between EtOAc $(100 \mathrm{~mL})$ and $2 \mathrm{~N} \mathrm{~K}_{2} \mathrm{CO}_{3}$ solution $(2 \times 50 \mathrm{~mL})$. The organic layer was washed with brine $(50 \mathrm{~mL})$, dried over magnesium sulfate, and evaporated under reduced pressure. The obtained residue was purified by silica gel column chromatography (10\% diethyl ether in dichloromethane) to afford compound $\mathbf{6 a}-\mathbf{q}$.

\section{Supporting Information}

\section{Supporting Information File 1 \\ Experimental section. \\ [http://www.beilstein-journals.org/bjoc/content/ supplementary/1860-5397-9-246-S1.pdf]}

\section{Acknowledgements}

The authors wish to thank the F.W.O [Fund for Scientific Research - Flanders (Belgium)] and the Research Fund of the University of Leuven (KU Leuven) for financial support. A.K. is thankful to EMA2experts (Erasmus Mundus Action 2, Lot 11 Asia: Experts) for providing a doctoral exchange scholarship, and D.D.V. is thankful to EMECW, lot 13 (Erasmus Mundus External Cooperation Window, Lot 13) for providing a doctoral scholarship. The authors thank Ir. B. Demarsin for HRMS measurements.

\section{References}

1. Ruijter, E.; Scheffelaar, R.; Orru, R. V. A. Angew. Chem., Int. Ed. 2011, 50, 6234-6246. doi:10.1002/anie.201006515

2. Ganem, B. Acc. Chem. Res. 2009, 42, 463-472. doi:10.1021/ar800214s

3. El Kaïm, L.; Grimaud, L. Mol. Diversity 2010, 14, 855-867. doi:10.1007/s11030-009-9175-3
4. Dömling, A.; Ugi, I. Angew. Chem., Int. Ed. 2000, 39, 3168-3210. doi:10.1002/1521-3773(20000915)39:18<3168::AID-ANIE3168>3.0.CO ;2-U

5. Dömling, A. Chem. Rev. 2006, 106, 17-89. doi:10.1021/cr0505728

6. Ramón, D. J.; Yus, M. Angew. Chem., Int. Ed. 2005, 44, 1602-1634. doi:10.1002/anie.200460548

7. Dömling, A.; Wang, W.; Wang, K. Chem. Rev. 2012, 112, 3083-3135. doi:10.1021/cr100233r

8. Shriri, M. Chem. Rev. 2012, 112, 3508-3549. doi:10.1021/cr2003954

9. Chen, Z.; Zheng, D.; Wu, J. Org. Lett. 2011, 13, 848-851. doi:10.1021/ol102775s

10. Tietze, L. F.; Rackelmann, N. Pure Appl. Chem. 2004, 76, 1967-1983. doi:10.1351/pac200476111967

11. Yang, J.; Xie, X.; Wang, Z.; Mei, R.; Zheng, H.; Wang, X.; Zhang, L.; Qi, J.; She, X. J. Org. Chem. 2013, 78, 1230-1235. doi:10.1021/jo302404v

12. Cheng, H.-G.; Lu, L.-Q.; Wang, T.; Yang, Q.-Q.; Liu, X.-P.; Li, Y.; Deng, Q.-H.; Chen, J.-R.; Xiao, W.-J. Angew. Chem., Int. Ed. 2013, 52, 3250-3254. doi:10.1002/anie.201209998

13. El Kaïm, L.; Grimaud, L.; Le Goff, X.-F.; Menes-Arzate, M.; Miranda, L. D. Chem. Commun. 2011, 47, 8145-8147. doi:10.1039/c1cc12236c

14. Bai, B.; Li, D.-S.; Huang, S.-Z.; Ren, J.; Zhu, H.-J. Nat. Prod. Bioprospect. 2012, 2, 53-58. doi:10.1007/s13659-012-0003-6

15. Lajiness, J. P.; Jiang, W.; Boger, D. L. Org. Lett. 2012, 14, 2078-2081. doi:10.1021/ol300599p

16. Fan, F.; Xie, W.; Ma, D. Org. Lett. 2012, 14, 1405-1407. doi:10.1021/ol3003496

17. Fürstner, A. Chem. Soc. Rev. 2009, 38, 3208-3221. doi:10.1039/b816696j

18. Dyker, G. Angew. Chem., Int. Ed. 2000, 39, 4237-4239. doi:10.1002/1521-3773(20001201)39:23<4237::AID-ANIE4237>3.0.CO ;2-A

19. Hashmi, A. S. K.; Hutchings, G. Angew. Chem., Int. Ed. 2006, 45 , 7896-7936. doi:10.1002/anie.200602454

20. Fürstner, A.; Davies, P. W. Angew. Chem., Int. Ed. 2007, 46, 3410-3449. doi:10.1002/anie.200604335

21. Gorin, D. J.; Sherry, B. D.; Toste, F. D. Chem. Rev. 2008, 108, 3351-3378. doi:10.1021/cr068430g

22. Jiménez-Núñez, E.; Echavarren, A. M. Chem. Rev. 2008, 108, 3326-3350. doi:10.1021/cr0684319

23. Li, Z. G.; Brouwer, C.; He, C. Chem. Rev. 2008, 108, 3239-3265. doi:10.1021/cr068434I

24. Arcadi, A. Chem. Rev. 2008, 108, 3266-3325. doi:10.1021/cr068435d

25. Hashmi, A. S. K.; Rudolph, M. Chem. Soc. Rev. 2008, 37, 1766-1775. doi:10.1039/b615629k

26. Rudolph, M.; Hashmi, A. S. K. Chem. Soc. Rev. 2012, 41, 2448-2462. doi:10.1039/c1cs15279c

27. Echavarren, A. M. Nat. Chem. 2009, 1, 431-433. doi:10.1038/nchem.344

28. Jiménez-Núñez, E.; Echavarren, A. M. Chem. Commun. 2007, 333-346. doi:10.1039/b612008c

29. Rudolph, M.; Hashmi, A. S. K. Chem. Commun. 2011, 47, 6536-6544. doi:10.1039/c1cc10780a

30. Hashmi, A. S. K. Angew. Chem., Int. Ed. 2010, 49, 5232-5241. doi:10.1002/anie.200907078

31. Ferrer, C.; Echavarren, A. M. Angew. Chem., Int. Ed. 2006, 45, 1105-1109. doi:10.1002/anie.200503484 
32. Ferrer, C.; Amijs, C. H. M.; Echavarren, A. M. Chem.-Eur. J. 2007, 13, 1358-1373. doi:10.1002/chem.200601324

33. Ferrer, C.; Escribano-Cuesta, A.; Echavarren, A. M. Tetrahedron 2009, 65, 9015-9020. doi:10.1016/j.tet.2009.08.067

34. Hashmi, A. S. K.; Yang, W.; Rominger, F. Angew. Chem., Int. Ed. 2011, 50, 5762-5765. doi:10.1002/anie.201100989

35. Hashmi, A. S. K.; Yang, W.; Rominger, F. Chem.-Eur. J. 2012, 18 , 6576-6580. doi:10.1002/chem.201200314

36. Chaładaj, W.; Corbet, M.; Fürstner, A. Angew. Chem., Int. Ed. 2012, 51, 6929-6933. doi:10.1002/anie.201203180

37. Loh, C. C. J.; Badorrek, J.; Raabe, G.; Enders, D. Chem.-Eur. J. 2011, 17, 13409-13414. doi:10.1002/chem.201102793

38. Lu, Y.; Du, X.; Jia, X.; Liu, Y. Adv. Synth. Catal. 2009, 351, 1517-1522. doi:10.1002/adsc.200900068

39. Xie, X.; Du, X.; Chen, Y.; Liu, Y. J. Org. Chem. 2011, 76, 9175-9181. doi:10.1021/jo2017668

40. Zhang, L. J. Am. Chem. Soc. 2005, 127, 16804-16805. doi:10.1021/ja056419c

41. Cera, G.; Crispino, P.; Monari, M.; Bandini, M. Chem. Commun. 2011, 47, 7803-7805. doi:10.1039/c1cc12328a

42. Cera, G.; Chiarucci, M.; Mazzanti, A.; Mancinelli, M.; Bandini, M. Org. Lett. 2012, 14, 1350-1353. doi:10.1021/ol300297t

43. Liu, Y.; Xu, W.; Wang, W. Org. Lett. 2010, 12, 1448-1451. doi:10.1021/ol100153h

44. Modha, S. G.; Kumar, A.; Vachhani, D. D.; Jacobs, J.; Sharma, S. K.; Parmar, V. S.; Van Meervelt, L.; Van der Eycken, E. V. Angew. Chem., Int. Ed. 2012, 51, 9572-9575. doi:10.1002/anie.201205052

45. Modha, S. G.; Kumar, A.; Vachhani, D. D.; Sharma, S. K.; Parmar, V. S.; Van der Eycken, E. V. Chem. Commun. 2012, 48, 10916-10918. doi:10.1039/c2cc35900f

46. Vachhani, D. D.; Galli, M.; Jacobs, J.; Van Meervelt, L.; Van der Eycken, E. V. Chem. Commun. 2013, 49, 7171-7173. doi:10.1039/c3cc43418d

47. Modha, S. G.; Mehta, V. P.; Van der Eycken, E. V. Chem. Soc. Rev. 2013, 42, 5042-5055. doi:10.1039/c3cs60041f

48. Kumar, A.; Vachhani, D. D.; Modha, S. G.; Sharma, S. K.; Parmar, V. S.; Van der Eycken, E. V. Eur. J. Org. Chem. 2013, 2288-2292. doi:10.1002/ejoc.201300132

49. Vachhani, D. D.; Sharma, A.; Van der Eycken, E. J. Org. Chem. 2012, 77, 8768-8774. doi:10.1021/jo301401q

50. Vachhani, D. D.; Mehta, V. P.; Modha, S. G.; Van Hecke, K.; Van Meervelt, L.; Van der Eycken, E. V. Adv. Synth. Catal. 2012, 354, 1593-1599. doi:10.1002/adsc.201100881

51. Vachhani, D. D.; Kumar, A.; Modha, S. G.; Sharma, S. K.; Parmar, V. S.; Van der Eycken, E. V. Eur. J. Org. Chem. 2013, 1223-1227. doi:10.1002/ejoc.201201587

52. Modha, S. G.; Trivedi, J. C.; Mehta, V. P.; Ermolat'ev, D. S.; Van der Eycken, E. V. J. Org. Chem. 2011, 76, 846-856 doi:10.1021/jo102089h

53. Modha, S. G.; Mehta, V. P.; Ermolat'ev, D. S.; Balzarini, J.; Van Hecke, K.; Van Meervelt, L.; Van der Eycken, E. V. Mol. Diversity 2010, 14, 767-776. doi:10.1007/s11030-009-9221-1

54. Kumar, A.; Li, Z.; Sharma, S. K.; Parmar, V. S.; Van der Eycken, E. V. Org. Lett. 2013, 15, 1874-1877. doi:10.1021/ol400526a

55. Mehta, V. P.; Modha, S. G.; Ruijter, E.; Van Hecke, K.; Van Meervelt, L.; Pannecouque, C.; Balzarini, J.; Orru, R. V. A.; Van der Eycken, E. V. J. Org. Chem. 2011, 76, 2828-2839. doi:10.1021/jo200251q
56. Peshkov, V. A.; Pereshivko, O. P.; Van der Eycken, E. V. Chem. Soc. Rev. 2012, 41, 3790-3807. doi:10.1039/c2cs15356d

57. Vachhani, D. D.; Sharma, A.; Van der Eycken, E. V. Angew. Chem., Int. Ed. 2013, 52, 2547-2550. doi:10.1002/anie.201209312

58. Modha, S. G.; Vachhani, D. D.; Jacobs, J.; Van Meervelt, L.; Van der Eycken, E. V. Chem. Commun. 2012, 48, 6550-6552. doi:10.1039/c2cc32586a

59. Donets, P. A.; Van Hecke, K.; Van Meervelt, L.; Van der Eycken, E. V. Org. Lett. 2009, 11, 3618-3621. doi:10.1021/ol901356h

60. Donets, P. A.; Van der Eycken, E. V. Synthesis 2011, 2147-2153. doi:10.1055/s-0030-1260057

61. Zhou, C.-Y.; Chan, P. W. H.; Che, C.-M. Org. Lett. 2006, 8, 325-328. doi:10.1021/ol052696c

62. Brand, J. P.; Waser, J. Angew. Chem., Int. Ed. 2010, 49, 7304-7307. doi:10.1002/anie.201003179

63. Vachhani, D. D.; Modha, S. G.; Sharma, A.; Van der Eycken, E. V. Tetrahedron 2013, 69, 359-365. doi:10.1016/j.tet.2012.10.019

64. Tubaro, C.; Baron, M.; Biffis, A.; Basato, M. Beilstein J. Org. Chem. 2013, 9, 246-253. doi:10.3762/bjoc.9.29

\section{License and Terms}

This is an Open Access article under the terms of the Creative Commons Attribution License

(http://creativecommons.org/licenses/by/2.0), which permits unrestricted use, distribution, and reproduction in any medium, provided the original work is properly cited.

The license is subject to the Beilstein Journal of Organic Chemistry terms and conditions:

(http://www.beilstein-journals.org/bjoc)

The definitive version of this article is the electronic one which can be found at: doi:10.3762/bjoc. 9.246 